\title{
EL MUSEO PEDAGÓGICO Y DEL NIÑO DE CASTILLA-LA MANCHA, UN EJEMPLO DE INTERACCIÓN CON SU ENTORNO SOCIAL
}

\section{The Pedagogic and Children Museum of Castilla-La Mancha, an example of interaction with their social environment}

\author{
Juan Peralta Juárez \\ Recepción: I2 de abril de 2016 \\ Envío a informantes: 14 de abril de 2016 \\ Aceptación definitiva: 5 de mayo de 2016
}

Director del Museo Pedagógico y del Niño de Castilla-La Mancha (Albacete)

Correo-e: museo.pedagogico@jccm.es; peraltaj@ono.com

Resumen: El Museo Pedagógico y del Niño de Castilla-La Mancha (conocido hasta ahora como Museo del Niño), además de contribuir a la custodia y difusión del patrimonio educativo de nuestra Comunidad es, sobre todo, un espacio abierto a todas las manifestaciones de la cultura material de la infancia y la adolescencia, es decir, un centro museístico que tiene en cuenta al niño en todas y cada una de sus diferentes manifestaciones culturales: la escuela, la familia, la calle y el trabajo. Además, el Museo del Niño (en adelante, MUNI) cumple también con otra de las funciones que debe tener todo museo pedagógico que es la de contribuir a la formación e innovación del profesorado, poniendo a su disposición un centro documental con todos los recursos necesarios para la planificación y desarrollo de actividades con alumnos y para la realización de trabajos de fin de carrera y de doctorado. Pero, sobre todo, el MUNI es un museo de y para la sociedad en la que está inmerso, principalmente porque permite al visitante -niño, joven o adulto- tener vivencias y recuerdos que le permitan recordar, sentir y vivir emociones de sus antepasados, de ellos mismos o de otros seres y otras culturas, lejanas o próximas a las suyas.

Palabras clave: Museo Pedagógico y del Niño; Castilla-La Mancha; entorno y sociedad.

Aвstract: The Pedagogic and Children of Castilla-La Mancha Museum, in addition to contributing to the custody and dissemination of educational heritage of our community is, above all, museum and society in which it is immersed, mainly because it allows 
EL MUSEO PEDAGÓGICO Y DEL NIÑO DE CASTILLA-LA MANCHA, UN EJEMPLO DE INTERACCIÓN CON SU ENTORNO SOCIAL JUAN PERALTA JUÁREZ

the -child, youth and adult have experiences and memories that allow you to remember, feel and experience emotions of their ancestors, themselves or other beings visiting and other cultures, distant or close to theirs.

Key words: The Pedagogic and Children Museum; Castilla-La Mancha; social environment.

\section{Consumir cultura museística, ¿̨una necesidad o una moda?}

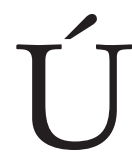

LTIMAMENTE, ES FRECUENTE VER GRANDES MASAS DE VISITANTES en determinados museos dispuestas a contemplar exposiciones que previamente han sido difundidas por todos los medios de comunicación, y muy especialmente por la televisión. Ir a un museo se ha convertido, pues, en algo más que una necesidad cultural, diríamos, que, en muchas de esas ocasiones, es un signo externo de distinción social, de estar a la última, de esnobismo. Así, las agencias de viajes organizan periplos turísticos en los que se incluye la visita a tal o cual museo, recientemente inaugurado, o a una exposición temporal de un pintor o escultor famosos, dándose la paradoja de que muchas de las obras que vamos a contemplar en esa exposición estrella están expuestas permanentemente en las salas del museo al que no solemos ir con relativa frecuencia, pudiéndolas ver sin aglomeraciones en otras fechas fuera del periodo excepcional de programación de esa exposición-espectáculo que tanto interés ha despertado.

Pero, en contraste con los museos tradicionales, lo que más llama la atención es la tendencia actual imperante en la creación o remodelación de algunos espacios museísticos, convirtiéndose en algo parecido a los parques de atracciones temáticos, en los que las nuevas tecnologías y el propio continente, es decir, el edificio, constituyen la parte fundamental del reclamo publicitario. Edificios diseñados por arquitectos de reconocido prestigio universal y módulos expositores basados en la utilización interactiva de los cinco sentidos son elementos imprescindibles para que un museo se convierta en museo-estrella, independientemente de la categoría o naturaleza de las obras que en él se exponen. Como dice la presidenta del Consejo Internacional de los Museos, «el visitante no va al museo sólo para aprender, sino también para distraerse y en busca de nuevas formas de seducción visual».

$\mathrm{Y}$, sin embargo, las peregrinaciones en masa a los museos forman parte de determinados ritos que ya en la Antigüedad se llevaban a cabo. Los romanos se convirtieron en los primeros coleccionistas de objetos, que previamente habían sido expoliados a los griegos. Así, el coleccionismo, mediante adquisición, donación o expoliación -como el caso que estamos describiendo-, ha sido en muchas ocasiones el origen de los antiguos museos, entendidos como lugares de almacenamiento y exposición de objetos que habían pertenecido a otros pueblos y culturas, objetos que había que exponerlos a la contemplación y curiosidad del pueblo para que admirasen el poderío y la grandeza de sus países.

Con el cristianismo, se exponen en las iglesias determinados objetos que habían pertenecido o habían estado real o supuestamente en contacto con Jesucristo, los apóstoles y otros mártires de la persecución romana. Así, se propició el que miles y miles de personas peregrinasen a las ciudades santas de Roma y Jerusalén para «poder admirar huesos y reliquias de un poder extraordinario para el creyente». 
EL MUSEO PEDAGÓGICO Y DEL NIÑO DE CASTILLA-LA MANCHA, UN EJEMPLO DE INTERACCIÓN CON SU ENTORNO SOCIAL JUAN PERALTA JUÁREZ

El carácter mítico, sagrado o de dominio que tenían los objetos expuestos en la Antigüedad se ve transformado en el Renacimiento, en donde el ser humano se convierte en el centro de todo el saber y, por lo tanto, todo aquello que ha sido realizado por él o ha estado en contacto con el mismo se ve investido de una capacidad de conocimiento que es necesario conocer. Pero, al mismo tiempo, el atesorar objetos antiguos o coetáneos a esa época era sinónimo de poder y prestigio. Las grandes colecciones de arte en el Renacimiento van unidas a grandes señores de la Iglesia o de la aristocracia y burguesía, como es el caso de los Médicis en Florencia.

Hasta ahora, lo que llevamos visto es que el concepto de museo en las Edades Antigua, Media y Moderna no se correspondía -como es obvio- con lo que es actualmente. Eran, sí, colecciones de objetos, dotados de carácter mítico, sagrado o de poder económico o social, pero siempre privados, bien de la Iglesia o de señores de la nobleza. Con la Ilustración, las colecciones de objetos antiguos se convierten en medios imprescindibles para la ciencia racional, para explicar el porqué y el cómo de determinados problemas científicos. Y, de esta manera, empiezan a abrirse al público, con la finalidad de enriquecer el conocimiento humano, de educar y enseñar, lema que es adoptado por la Revolución francesa, quien abre al público los tesoros acumulados por reyes y nobleza.

En el siglo XIX surgen los Museos Nacionales, en los que se exalta la nación a través de todos aquellos objetos artísticos que pertenecen a su larga trayectoria como pueblo y que la hace distinta de las demás. A lo autóctono había que unir lo conseguido en las campañas militares que como potencias llevaban a cabo algunos países europeos en África, Asia y América, principalmente. Surgen de esta manera los museos como señas de identidad cultural nacional al mismo tiempo que signo de poder y dominio. Los grandes museos nacionales de Francia y el Reino Unido, por citar sólo dos casos, son ejemplos vivos de lo que estamos diciendo: Napoleón, por un lado, y los conquistadores británicos, por otro, expoliaron riquezas artísticas de los antiguos Egipto, Grecia, Mesopotamia, Irán, Irak y la India, entre otros muchos lugares, trasladándolas a sus naciones y exponiéndolas en el British Museum o el Louvre para admiración y sorpresa de sus respectivos pueblos.

Así, el museo, como espacio expositor de riquezas artísticas, ha desempeñado diversos papeles según la ideología del poder dominante: lugares sagrados en los que adorar objetos pertenecientes a héroes y santos; espacios para divulgar el conocimiento científico; galerías en las que se mostraba el poder del mecenas de la nobleza o de la monarquía; palacios en los que se ponía de manifiesto la grandeza e identidad cultural del Imperio... En la actualidad, los museos, tras haber atravesado una etapa de cierta atonía, parece que vuelven a recobrar nuevos bríos, atrayendo el interés de gran parte de los ciudadanos, sobre todo de aquellos que ocupan un estatus social y cultural medio-alto. Pero, para conseguirlo han tenido que cambiar su naturaleza, pasando de ser espacios estáticos a espacios comprensivos.

\section{Del museo tradicional al museo comprensivo}

«La acepción que tiene actualmente el término museo dista mucho del concepto tradicional. El museo es hoy una institución al servicio de la sociedad; queda ya lejos el museo donde se veneran, admiran y conservan una serie de piezas sin que exista 
EL MUSEO PEDAGÓGICO Y DEL NIÑO DE CASTILLA-LA MANCHA, UN EJEMPLO DE INTERACCIÓN CON SU ENTORNO SOCIAL JUAN PERALTA JUÁREZ

entre lo exhibido y el público visitante una comunicación, un diálogo». No sé si estaremos o no de acuerdo con esta afirmación que hacía M. ${ }^{a}$ Carmen Farré, del Museo de Arte de Cataluña, allá por i974. Pero la realidad es que, salvo excepciones, continúa habiendo museos en los que la gente no encuentra aliciente para ir a ellos. Para muchas personas, con un nivel cultural medio, los museos constituyen espacios muertos, que no merece la pena visitar, y se da la paradoja, como se ha dicho al principio, de que esas mismas personas se inscriben en peregrinaciones mítico-culturales a museos que han sido previamente publicitados con costosísimas campañas de divulgación en radio, prensa y televisión. ¿Cómo se explica este contrasentido? ¿Qué es lo que mueve a esas personas a desplazarse cientos de kilómetros a visitar esos espacios expositivos cuando normalmente no conocen los museos que tienen en su entorno? Como se ha dicho anteriormente, inciden varios aspectos: uno, la cultura se ha convertido en un producto más del mercado, que hay que consumir mediatizados por los estrategas publicistas; dos, la cultura museística ha entrado en los grandes circuitos culturales y, al igual que las visitas a ciudades monumentales, se programan visitas a grandes museos nacionales de arte, arqueología, ciencia, etc.; tres, los museos se están convirtiendo en una especie de parques tecnológicos en los que prima el espectáculo por encima de lo demás. Para el profesor Santana, «el consumo museístico se transforma en una actividad social: actividad social articulada en torno a la escuela; actividad social en torno al turismo de masas o actividad social en torno al prestigio intelectual de determinadas élites».

\section{Las interrelaciones entre los agentes de la sociedad educadora}

Últimamente se ha «puesto de moda» en los medios de comunicación dar noticias sobre la violencia escolar en las aulas. Parece como si de la noche a la mañana se hubiesen todos puesto de acuerdo: prensa, radio y televisión, para dar noticias de este tipo. Noticias que, en gran medida, vienen magnificadas por los propios medios y sirven de caja de resonancia para las siguientes. ¿Acaso no ha habido violencia o agresividad escolar en otras épocas? Si indagamos un poco en la historia de la educación, vemos que en todos los momentos y en todos los lugares el tema de la disciplina escolar ha sido algo que ha preocupado a todos los educadores. No es que ahora los niños sean más agresivos que en el pasado, lo que ocurre es que el medio en el que se desenvuelve su vida cotidiana difunde y potencia multitud de mensajes que promueven el materialismo, el egoísmo, el individualismo y la agresividad como instrumentos para vivir en este planeta y abrirse camino en la jungla humana.

De las 24 horas que tiene el día, un niño pasa la mayor parte del tiempo, cuando no está en el aula, en casa solo delante del televisor o jugando con la videoconsola. Por lo tanto, el medio audiovisual, que en gran parte es un medio agresivo y violento, está alimentando al niño con contenidos que son verdaderas armas contra la solidaridad y la convivencia, contra el respeto a nuestros semejantes, contra el valor del trabajo y las cosas bien hechas. Esos medios que propician el consumismo, el triunfo fácil, el mal gusto, la insolidaridad, la mentira... llegan al niño con relativa facilidad y sin ningún tipo de control por parte de la Administración, de la sociedad y de los padres. Ante ese desarme, ante ese dejar hacer, padres y responsables administrativos recurren a la escuela, a los maestros, como tabla de salvación, y así, de esta manera, se sobrecarga 
EL MUSEO PEDAGÓGICO Y DEL NIÑO DE CASTILLA-LA MANCHA, UN EJEMPLO DE INTERACCIÓN CON SU ENTORNO SOCIAL JUAN PERALTA JUÁREZ

el currículo y el horario escolar con infinidad de temas transversales, actividades complementarias, días de, etc., etc., como medio de salvar al niño, que se encuentra indefenso ante el mundo que le rodea. Hasta que los maestros no sólo no son capaces de poder abordar ellos solos la solución del problema, sino que esa «agresividad», que los niños están viviendo día a día, la sufren en carne propia los educadores.

En el origen de los tiempos, el niño recibía los escasos conocimientos que necesitaba para ser adulto en el seno de la propia familia. Conforme el mundo rural se fue urbanizando y la división del trabajo se generalizó, la educación se fue convirtiendo en algo más complejo, a la que había que dedicar más horas por parte de personal más especializado, ya que los padres no sabían o no tenían tiempo para ejercer de maestros con sus hijos. Así, pues, la escuela es una invención de la sociedad como instrumento para culturizar y educar de una manera más o menos homogénea y controlada a los niños.

A lo largo de muchos años, los padres y el resto de la sociedad han dejado sobre la escuela y los educadores la ingente tarea de la instrucción y educación de sus hijos, hasta que ha llegado el momento en que han saltado todas las alarmas y psicólogos, padres, educadores y Administración han dicho en voz alta lo que ya se sabía desde siempre: EDUCAR ES TAREA DE TODOS.

Como todos los padres saben, la educación es un proceso complejo que no consiste en transmitir y asimilar conocimientos. Si fuese eso únicamente, las máquinas, muchas veces, harían su trabajo mucho mejor que los maestros. Educar, pues, requiere desarrollar en el niño y la niña actitudes, habilidades y destrezas de todo tipo, pero básicamente aquellas que contribuyen a que el individuo sea autónomo, libre, responsable y solidario con sus semejantes y, por tanto, con el mundo en el que vive.

Profesores, padres e instituciones públicas y privadas tienen, mejor dicho, tenemos que colaborar en construir un medio físico, cultural, histórico y natural que potencie los valores morales del ser humano y que ponga al servicio de los escolares y educadores todos los recursos para lograr un mundo en el que el conflicto se resuelva de forma dialogada y pacífica. Para ello, como sabemos que los niños aprenden a través de modelos, la ciudad -con todos sus recursos- debe ser un ejemplo de convivencia para los escolares y un recurso fundamental para desarrollar todas sus potencialidades humanas de forma armónica y pacífica.

Es, pues, en la ciudad donde se dan esas interrelaciones entre todos los agentes educativos, no solamente humanos, sino físicos y culturales. El concepto de ciudad educadora, tan en boga hoy en día, no debe referirse a cómo solucionar el problema de los tiempos escolares, es decir, de los horarios. No es, o no debería serlo, una agenda con multitud de actividades extraescolares como forma de tener ocupados a los escolares la mayor parte del tiempo. No, ciudad educadora implica abrir la escuela a la ciudad y proyectar la escuela en la ciudad. Bajo el primer enfoque, la ciudad se convierte en objeto y agente de aprendizaje; bajo el segundo enfoque, la ciudad se convierte en fin educativo y medio para hacer ciudadanos libres. Aprender para la ciudad supone que la ciudad es portadora y transmisora de valores, lo que exige educar en las escuelas para que los niños se conviertan en ciudadanos que participen, de manera crítica y constructiva, en la mejora de la ciudad.

La ciudad, con sus parques, museos, centros comerciales, centros de transporte, plazas, calles..., es el lugar idóneo para que el niño aprenda pautas de comportamiento y normas básicas de convivencia, para que valore las situaciones de riesgo y peligro de determinadas actitudes, para que aprenda y ejercite todo tipo de valores 
EL MUSEO PEDAGÓGICO Y DEL NIÑO DE CASTILLA-LA MANCHA, UN EJEMPLO DE INTERACCIÓN CON SU ENTORNO SOCIAL JUAN PERALTA JUÁREZ

sociales. La ciudad, en definitiva, es lugar idóneo para que los niños trabajen y vivencien en el día a día valores como la justicia, la paz y la solidaridad, que, en suma, son valores propios de la Educación para la Paz.

\section{Los museos pedagógicos, recursos básicos para la sensibilización de la comunidad en el contexto de una ciudad educadora}

Los museos pedagógicos son, por su propia naturaleza, un recurso fundamental para la educación y sensibilización de la sociedad. Son, pues, una pieza fundamental del concepto de ciudad educadora, entendido esto último como aquella en cuyo seno se dan e interrelacionan procesos educativos formales (escuelas, institutos, universidad, etc.); no formales (actividades de tiempo libre y de otro tipo organizadas por asociaciones de padres, vecinos o ayuntamiento), e informales, entendiendo por este último concepto todo aquello que aporta información al individuo y que, como tal, lo está mediatizando (medios de comunicación y publicidad). La ciudad como agente de educación tiene un carácter abierto y dinámico.

Aprender en la ciudad es tanto como admitir que la ciudad es portadora y transmisora de valores, siendo la escuela la que debe aprovechar esos valores para la educación de los jóvenes ciudadanos en la línea que permita rescatar o potenciar aquellos comportamientos humanos que incidan en el individuo de forma positiva.

La ciudad, en donde se dan las interrelaciones sociales, se hace día a día con el comportamiento cívico de sus ciudadanos, que a su vez inician su formación como tales desde la más tierna infancia en el seno de la institución escolar y familiar. Por ello, la escuela junto con su entorno, es decir, la ciudad, deben favorecer una educación en la que prime una determinada escala de valores que favorezcan el desarrollo de todos sus ciudadanos en armonía y paz con su medio. Pero, ¿cuáles deben ser esos valores? La solidaridad frente a la insolidaridad; la convivencia pacífica frente al vandalismo de las «tribus urbanas»; el uso y respeto de lo público frente al deterioro del mismo. La formación del ciudadano es según Molás Batllori un problema ineludible para los sectores democráticos que adoptan la escuela como instrumento activo para formar ciudadanos capaces de optar racionalmente, sin necesidad de pastores ni de autoridades omniscentes. La escuela, como vía principal de socialización para la formación de ciudadanos, convirtió la enseñanza y la formación en educación. El civismo o la urbanidad, como conjunto coherente de criterios para moverse en colectividad, fueron introducidos como materia escolar al mismo tiempo que la aritmética o la lengua. La escuela, pues, se convirtió de esta manera en el gran instrumento de socialización y de aprendizaje.

En resumen, si admitimos que la ciudad, con todos sus recursos, debe aprovecharse por parte de los educadores para trabajar diversas actitudes, valores y normas que hagan del escolar un ciudadano conocedor del pasado histórico de la misma y responsable en sus actitudes para lograr un mundo mejor, los museos y otros centros culturales son recursos que se deben aprovechar al máximo para conseguir dichos fines.

\section{El Museo Pedágogico y del Niño y su proyección en el entorno social}

El Museo Pedagógico y del Niño de Castilla-La Mancha -conocido como Museo del Niño- ofrece a la familia, a los educadores y a los escolares un conjunto de recursos 
EL MUSEO PEDAGÓGICO Y DEL NIÑO DE CASTILLA-LA MANCHA, UN EJEMPLO DE INTERACCIÓN CON SU ENTORNO SOCIAL JUAN PERALTA JUÁREZ

que facilitan la formación de valores en los alumnos como la empatía, la solidaridad, la convivencia, el entendimiento y el respeto por otras formas de vida y cultura. Así, el visitante puede conocer, a través de los materiales expuestos y las diversas actividades organizadas, cómo era la vida de los niños en los siglos xIx y xx en diferentes ámbitos de convivencia: la familia, la calle y la escuela; además de conocer los problemas actuales que afectan a muchos millones de niños en el mundo en la sección titulada «Infancias Robadas».

Este museo, en su carácter más amplio, es un verdadero museo etnográfico, ya que es el lugar en donde se expone la vida y cultura de un pueblo a través de los objetos cotidianos que los niños han usado a lo largo del tiempo en el hogar, en la calle y en la escuela. Un museo pedagógico es un museo del pueblo y para el pueblo, convirtiéndose en un instrumento eficaz para el rescate y difusión de las señas de identidad de ese pueblo. Este tipo de museos tienen la ventaja -sobre otros- que son «cercanos», o sea, familiares, para todos aquellos que los visitan, ya que los materiales expuestos forman o han formado parte de sus vidas. Los objetos expuestos son fácilmente comprensibles, sobre todo si están contextualizados en espacios que recreen la vida cotidiana, algo que no suele ocurrir con otros museos. Y son, además, museos que contribuyen al rescate de las señas de identidad de la sociedad a la que representan y en la que están inmersos.

En coherencia con lo expresado en el párrafo anterior, este museo, desde su fundación en 1987, ha tenido como objetivos básicos los siguientes:

I) Rescatar, conservar y difundir el patrimonio etnográfico de la infancia en todos sus ámbitos culturales: la familia, la calle y la escuela, principalmente.

2) Implicar a los diferentes núcleos sociales: alumnado, profesorado, familias e instituciones relacionadas con la educación y protección social de la infancia en aquellas actividades que realice el museo en el ámbito de su dinamización sociocultural.

Así, para conseguir ambos objetivos, el Museo ha llevado a cabo diversas tareas encaminadas a insertarse en la sociedad y devolver a la misma lo que ésta ha dado al museo representado en los múltiples objetos en él depositados. Para ello, los responsables de esta institución museística ofrecen a la comunidad:

a) El Cedohife (Centro de Documentación Histórica de la Infancia, la Familia y la Escuela), con más de 15.000 documentos, en soporte papel y audiovisual, clasificados en diez bases de datos en soporte informático. Así, el Museo del Niño tiene una de las pocas bases de datos que existen en España sobre la infancia y la familia, denominada oLIVER, utilizando para ello la poderosa herramienta de Knosys 2004, que es un sistema de gestión electrónica documental totalmente visual orientado a bases de datos y gestión de objetos. OLIVER agrupa diferentes documentos clasificados en los siguientes temas: i) Abandono del hogar; 2) Adopción de niños; 3) Delincuencia infantil y juvenil; 4) Derechos del Niño; 5) Drogadicción infantil y juvenil; 6) Educación y ocio infantil y juvenil; 7) Esclavitud infantil; 8) Hambre y pobreza en los niños; 9) La escolarización en el mundo; Io) La infancia en el mundo desarrollado; II) Los niños de la calle; I2) Maltrato infantil; I3) Mendicidad y pobreza infantil; I4) Niñas-madre; I5) Niños en la guerra; I6) Niños mimados; 17) Niños-soldado; ı8) Pornografía y 
EL MUSEO PEDAGÓGICO Y DEL NIÑO DE CASTILLA-LA MANCHA, UN EJEMPLO DE INTERACCIÓN CON SU ENTORNO SOCIAL

JUAN PERALTA JUÁREZ

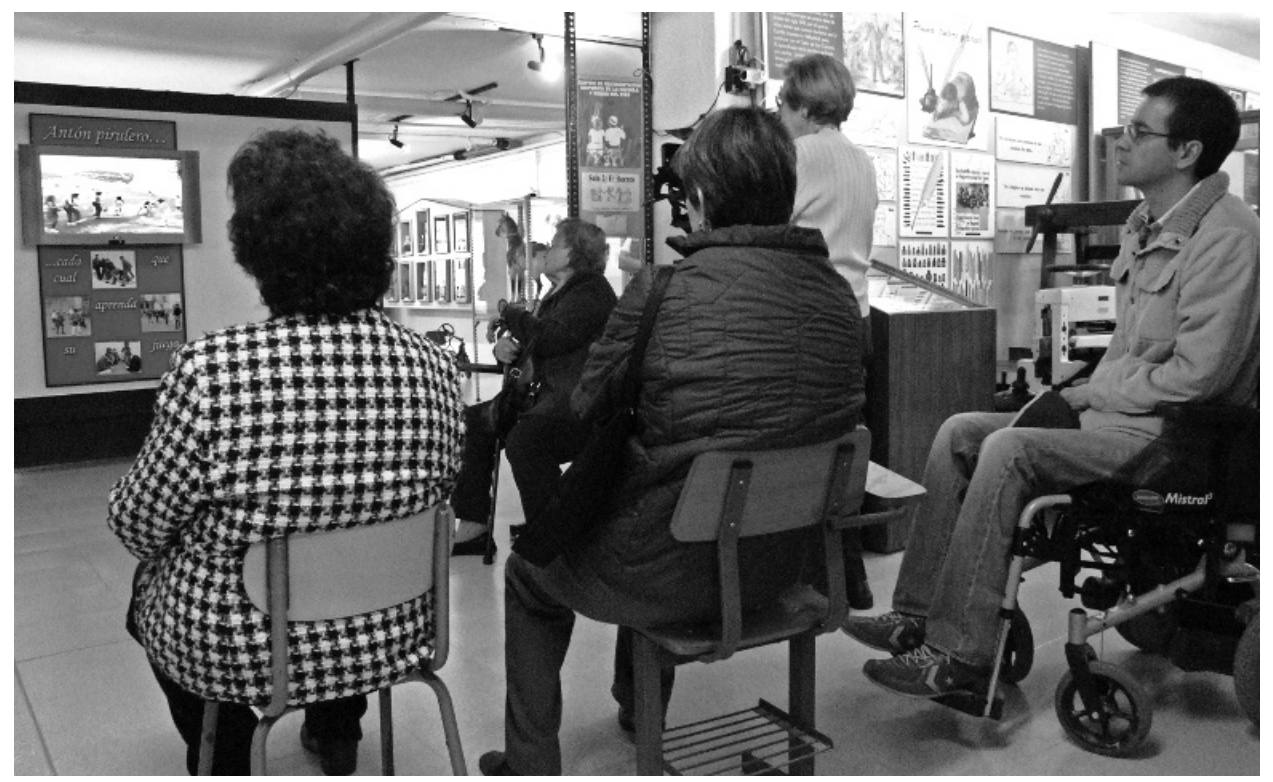

Figura i. Actividad en el museo con personas de un Centro de Mayores.

prostitución infantil; 19) Protección social de la infancia; 20) Publicidad infantil: usos y abusos; 2I) Trabajo infantil; 22) Tráfico de niños; 23) Violencia infantil y juvenil, y 24) Otros temas.

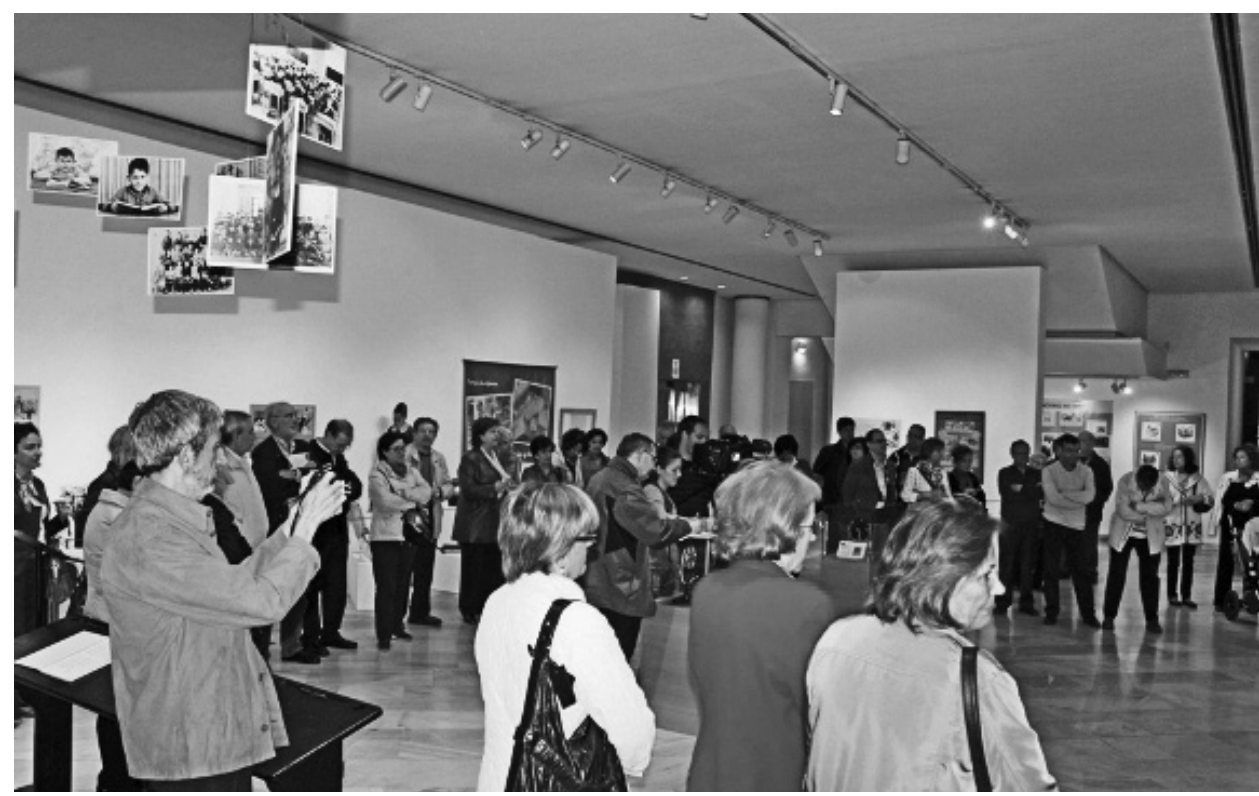

Figura 2. Acto social realizado en el Museo Municipal de Albacete, organizado por el Museo del Niño. 
EL MUSEO PEDAGÓGICO Y DEL NIÑO DE CASTILLA-LA MANCHA, UN EJEMPLO DE INTERACCIÓN CON SU ENTORNO SOCIAL JUAN PERALTA JUÁREZ

b) Historias de Vida. Documentos audiovisuales sobre la familia en el siglo xx. Materiales que sirven a los investigadores y estudiosos del tema para la realización de trabajos de investigación sobre las fuentes orales como recurso para el estudio de la vida cotidiana en el pasado. Hasta el momento se han editado cuatro documentales con la colaboración de instituciones como las Universidades Populares y los Centros de Mayores (Figura I).

c) Ciclos de conferencias, exposiciones y proyección de películas sobre dichos temas, a los que son invitados colectivos sociales de la ciudad (Figura 2).

d) Edición de publicaciones en donde se dan a conocer trabajos de investigación sobre la historia de la educación, la infancia y la familia (Figura 3).

Es decir, el Museo Pedagógico y del Niño devuelve a la sociedad, a la que se debe, el fruto de sus trabajos de recopilación e investigación, a través de actividades realizadas no solamente con el alumnado de los distintos centros escolares de la Comunidad, sino también con otros colectivos sociales a los que se les invita a realizar, entre otras actividades, Talleres de la Memoria, gracias a los cuales, y ante la presencia de objetos que formaron parte de sus vidas, los mayores rememoran sus años de infancia, recuerdos que quedan «custodiados» en el Centro de Documentación para que las generaciones venideras sepan y valoren cómo era la vida cotidiana de sus antepasados.

Resumiendo, a lo largo de sus veintinueve años de vida, este museo ha gozado del reconocimiento de la comunidad y de los medios de comunicación, que han sido los que han

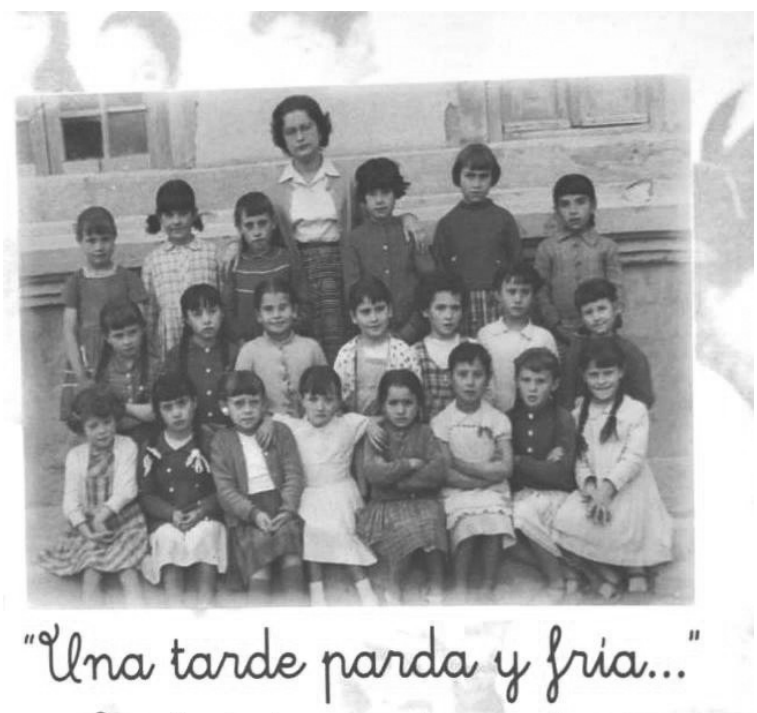

Antalogia de recuerdas escalares reclamado reiteradamente a la

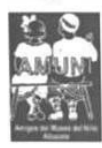

Figura 3. Publicación del Museo del Niño.

Administración soluciones para los muchos problemas que tenía esta institución. Y lo hacían, porque consideraban que el museo es algo fundamental para salvaguardar nuestra propia historia, la historia del pueblo, la historia de los que «no tienen historia», la de aquellos niños, maestros, padres, abuelos que iban a la escuela, que trabajaban en el campo o en el taller, que jugaban en la calle, que contaban historias al amor de la lumbre, que se calentaban en los fríos inviernos con una simple lata de ascuas... 
EL MUSEO PEDAGÓGICO Y DEL NIÑO DE CASTILLA-LA MANCHA, UN EJEMPLO DE INTERACCIÓN CON SU ENTORNO SOCIAL JUAN PERALTA JUÁREZ

\section{Bibliografía}

Ayuntamiento de Barcelona (1990) La ciudad y la ciudadanía democrática. Barcelona: Ayuntamiento de Barcelona.

Farré-Sanpera, M. ${ }^{a}$ Carme (1975) Aprender en el museo. Cuadernos de Pedagogía, n. ${ }^{\circ} 2$. Gesché-Knoning, Nicole «Museos y enseñanza de las Ciencias Sociales». Perspectivas de futuro. Gregorovius, F. (1982) Roma y Atenas en la Edad Media. México: FCE.

Santacana, Joan Museos, ¿al servicio de quién? Revista Iber, I5, 40 y 44. 